\title{
Autonomic dysfunction in gastrointestinal motility disorders
}

\author{
A E Bharucha, M Camilleri, P A Low, A R Zinsmeister
}

\begin{abstract}
The records of 113 consecutive patients with a suspected gastrointestinal motility disorder referred between January 1988 and July 1991 were retrospectively reviewed. The aims were to identify the prevalence of autonomic dysfunction in those with or without associated neurological disease and to determine the diagnostic value of testing for autonomic dysfunction. All patients had gastrointestinal manometry ( 3 hours fasting, 2 hours fed), 94 of 113 underwent testing of sympathetic adrenergic and cholinergic function and cardiovagal cholinergic function. All tests were scored in a standard manner. There was a significant $(p<0.05)$ but modest $(r=0.28)$ rank correlation between autonomic and motility scores. This correlation was stronger $(r=0.67$, $p=0.01)$ in diabetic patients. The number of patients in each group with autonomic dysfunction was as follows: irritable bowel syndrome nine of 33 , idiopathic upper gastrointestinal dysmotility six of 21 , diabetes mellitus nine of 13, identified non-diabetic neurological syndromes six of nine, postvagotomy or abdominal surgery three of 11 , and myopathic pseudo-obstruction two of seven. Autonomic testing is useful in the assessment of autonomic involvement outside the gastrointestinal tract. Logistic discriminant analysis showed that autonomic function testing did not add to the diagnostic value of motility tests in distinguishing between patients with and without irritable bowel syndrome, although a slight improvement was indicated for identifying neuropathic dysmotilities. Thus, the aetiological role of general autonomic dysfunction in irritable bowel syndrome and idiopathic and postvagotomy dysmotilities deserves further study. The addition of autonomic function tests does not add substantially to the diagnostic accuracy of clinical, radiological, endoscopic, and manometric techniques in most patients referred for evaluation of a suspected motility disorder.

(Gut 1993; 34: 397-401)
\end{abstract}

Gastroenterology Research Unit, Department of Neurology and Section of Biostatistics, Mayo Clinic and Mayo Foundation, Rochester, MN 55905, Roch

A E Bharucha

M Camilleri

P A Low

A R Zinsmeister

Correspondence to:

Dr M Camilleri,

Gastroenterology

Research Unit, Mayo Clinic,

Rochester, MN 55905, USA.

Accepted for publication

31 August 1992 manifestations suggestive of gastrointestinal dysmotility. ${ }^{12}$ These disturbances may affect all levels of the pathways (sympathetic and parasympathetic) supplying the digestive tract, from the brain to the postganglionic fibre. Autonomic function abnormalities have also been documented in patients with functional gastrointestinal disorders. ${ }^{3-5}$ Experimental models of gut motor function suggest a predominant modulatory role for the extrinsic nervous system, with primary control being exerted by the enteric nervous system. ${ }^{6}$ Thus, it is not clear how significantly the autonomic dysfunction impacts on gastrointestinal dysmotility and the development of functional gut disorders.

In addition, the prevalence of autonomic dysfunction among patients with gastrointestinal dysmotility remains unknown. A high prevalence of autonomic dysfunction among patients with or without known neurological disease would suggest that testing of autonomic function may be a useful alternative to the current invasive tests in the assessment of motility disturbances of the digestive tract.

Our aims were twofold: firstly to determine the prevalence of autonomic dysfunction in patients with suspected gastrointestinal dysmotility, with or without associated neurological disorders; and secondly, to assess the diagnostic value of testing autonomic function in these patients.

\section{PATIENTS}

We retrospectively reviewed the records of 113 consecutive patients with suspected motility disorders of the digestive tract referred to a single gastroenterologist (MC) at a tertiary referral clinic between January 1988 and July 1991. In all patients a detailed medical history was taken and physical examination, exclusion of mechanical obstruction or mucosal disease by endoscopy or barium radiology, and blood and urine tests include haematology and chemistry panels, erythrocyte sedimentation rate, antinuclear antibody, a serological test for syphilis (rapid plasma reagin), serum vitamin B12 and folate, serum thyroxine, and urine analysis were undertaken. A chest $x$ ray was also performed in all patients. Other investigations were done as deemed necessary on clinical grounds. A combination of clinical, radiological, endoscopic, and manometric criteria were used to group patients into diagnostic categories. Autonomic reflex and motility investigations (described below) were performed when the patients had been off all medications except insulin for at least 48 hours. All results were compared with data from healthy controls who had been studied in an identical manner in the respective laboratories at the Mayo Clinic during the previous 5 years.

\section{GASTROINTESTINAL MANOMETRY}

Gastric and small bowel pressure profiles were assessed by pneumohydraulic perfusion manometry. ${ }^{7}$ Motility was studied for 3 hours during fasting and 2 hours after ingestion of a solidoesophageal motility was evaluated using the liquid $535 \mathrm{kcal}$ meal. At the end of the study, 
station pull through method with wet $(1 \mathrm{cc})$ water swallows.

One of us (MC) had prospectively assessed all the manometric recordings and compiled standardised reports on them. In this analysis, patterns of the gastric and small bowel pressure profiles are analysed, with particular attention to the abnormalities recorded in previous reports of gastrointestinal motility in health and disease from this and other laboratories. ${ }^{7}$ These patterns include the following:

(a) An abnormal configuration and propagation of phase III of the migrating motor complex $(M M C)^{8}$ (the presence of $\geq 3 \mathrm{MMCs}$ during the 3 hour fasting period in patients with previous vagotomy or partial gastrectomy was also considered abnormal)."

(b) The presence of fasting or postprandial bursts of high amplitude phasic activity associated with a tonic elevation of baseline pressure. ${ }^{78}$

(c) Patterns suggestive of mechanical obstruction, such as persistent postprandial minute clustered contractions ${ }^{10}$ or simultaneous prolonged contractions."

(d) Postprandial antral hypomotility ${ }^{7}$ and pylorospasm, or a pattern of prolonged tonic and phasic pressure activity of the pylorus, usually associated with antral hypomotility. ${ }^{12}$

(e) Failure of the small bowel pressure profile to change from a fasting to a fed pattern, and this included the recurrence within 90 minutes of ingestion of the meal of the MMC.

(f) A pattern of minute clustered contractions associated with pain. ${ }^{1314}$

A semiquantitative assessment of the motility tracings was achieved using the scoring system

TABLE I Scoring system for upper gastrointestinal motility

\begin{tabular}{|c|c|}
\hline $\begin{array}{l}\text { Fasting: } \\
\text { Propagation of MMC }\end{array}$ & $\begin{array}{l}\text { Score if abnormal } \\
1 \cdot 5\end{array}$ \\
\hline If previous gastric surgery, $\geq 3 \mathrm{MMCs} / 3$ & \\
\hline hours & 1.5 \\
\hline $\begin{array}{l}\text { If no gastric surgery, absence of antral } \\
\text { component of } M M C\end{array}$ & 0.5 \\
\hline $\begin{array}{l}\text { Bursts/clusters associated with pain or tonic } \\
\text { elevation of baseline pressure }\end{array}$ & 1.5 \\
\hline If no MMCs, duodenojejunal hypomotility & 1.5 \\
\hline $\begin{array}{l}\text { Postprandial: } \\
\text { Antral hypomotility } \\
\text { Pylorospasm }\end{array}$ & $\begin{array}{l}\text { Score if abnormal } \\
3 \cdot 0 \\
0 \cdot 5\end{array}$ \\
\hline $\begin{array}{l}\text { Duodenojejunal incoordination or lack of } \\
\text { fed pattern }\end{array}$ & 1.0 \\
\hline Bursts/clusters & 1.5 \\
\hline $\begin{array}{l}\text { MMC like activity within } 90 \text { minutes of } \\
\text { end of meal }\end{array}$ & 1.5 \\
\hline
\end{tabular}

The maximum score of $11 \cdot 0$, in a subject who has not had prior gastric surgery, is possible if all of the italicised scores in the above table are scored. For this subject, an absolute score $\geq 3$ is abnormal. $\mathrm{MMC}=$ migrating motor complex.

TABLE II Scoring system for autonomic dysfunction

Sudomotor function (sympathetic cholinergic): sweating assessed at three sites:

$1=$ minor abnormality

$2=2$ sites reduced or 1 site absent

$3=3$ sites reduced or 2 sites absen

Cardiovagal function: cardiac response to deep breathing and Valsalva manoeuvre;

$1=$ borderline or midly abnorma

$2=$ moderate abnormality of one test

$3=$ both tests definitely abnormal

Sympathetic adrenergic function orthostatic hypotension, blood pressure (BP) responses during Valsalva manoeuvre:

(BP) responses during Valsalva manoeuvre:

$1=$ mild abnormality of indices during Valsalva manoeuvre

$2=$ no orthostatic hypotension, not abnormal Valsalva respon

$\mathrm{mm} \mathrm{Hg}$ ) +abnormal Valsalva responses shown in Table I. The weighting factors were based on previous experience of one investigator (MC) who has assessed over 2000 such tracings in patients with suspected motility disorders in our laboratory. A motility score of $\geq 3$ was considered abnormal for subjects who completed the entire 3 hour fasting and 2 hour postprandial study and ingested at least $400 \mathrm{kcal}$ of a mixed meal. The maximum score was 11 , which was used as the denominator to calculate the relative abnormality score for the motility test. For those patients who were unable to eat the meal or whose antrum had been excised at previous surgery, the maximum denominator was adjusted before computing the relative motility score. Clusters of small bowel contractions that were unassociated with pain or a failure to record any MMCs during the 3 hour fasting period were not considered abnormal.

\section{AUTONOMIC FUNCTION TESTS}

The following assessments of autonomic function were performed as previously described ${ }^{2}$ in 94 of the 113 patients who, for at least 48 hours, had been off all medications that could influence the test. One investigator (PAL) evaluated the results of the following tests:

(i) Supine and standing blood pressure, as a test of general sympathetic adrenergic function.

(ii) Heart rate and blood pressure responses to the Valsalva manoeuvre; a comparison of the ratio of the longest (usually in phase IV) to the shortest (usually in phase II) intervals between successive $R$ waves were measured on the electrocardiogram (ECG). In phase II, forced expiration against a closed glottis results in reduction in venous return, blood pressure, and cardiac output. A compensatory tachycardia normally serves to maintain cardiac output and can be detected by a shortening of the interval between successive QRS complexes on the ECG. During phase IV, the glottis is open, venous return and cardiac output are restored, with overshoot of the blood pressure and compensatory bradycardia. While the test predominantly evaluates sympathetic adrenergic function, it is also dependent on intact vagal innervation.

(iii) The sudomotor axon reflex test is a quantitative evaluation of the latency, output and duration of sweating following iontophoresis of acetylcholine into the skin of the forearm, leg (proximal and distal), and foot. This test evaluates postganglionic cholinergic sympathetic function.

(iv) Heart rate responses to deep breathing, a test of vagal cholinergic function.

Results of autonomic function tests were compared with normal data, matched for age and sex. The scoring system used is shown in Table II. An a priori cut off of $\geq 3$ was used to identify autonomic dysfunction in each patient. A relative autonomic dysfunction score was also computed for each patient using the maximum raw score of 9.

\section{STATISTICAL ANALYSIS}

A logistic discriminant analysis ${ }^{19}$ using both relative autonomic function and motility scores of all patients, except those with idiopathic 
TABle III Patient diagnoses

\begin{tabular}{lc}
\hline & Noof \\
Diagnosis & patients \\
\hline Irritable bowel syndrome: & 39 \\
Idiopathic upper gastrointestinal dysmotility: & 23 \\
Chronic idiopathic intestinal pseudo-obstruction & 18 \\
Idiopathic gastroparesis & 5 \\
Diabetes mellitus: & 13 \\
Type I & 11 \\
Type II & 2 \\
Non-diabetic neurological syndromes: & 9 \\
Paraneoplastic & 3 \\
Post-radiation & 2 \\
Post-viral dysautonomia & 2 \\
Porphyria & 1 \\
Motor neurone disease & 1 \\
After vagotomy/abdominal surgery: & 17 \\
Vagal damage & 4 \\
Billroth I & 2 \\
Roux-en-Y & 8 \\
Other abdominal surgery & 3 \\
Myopathic: & 12 \\
Progressive systemic sclerosis & 6 \\
Hollow visceral myopathy & 4 \\
Polymyositis & 1 \\
Mixed myopathic disorder & 1 \\
\hline
\end{tabular}

TABLE IV Prevalence of abnormal autonomic function $(A F)$

\begin{tabular}{|c|c|c|c|}
\hline Diagnosis & $\begin{array}{l}\text { Noof } \\
\text { patients }\end{array}$ & $\begin{array}{l}\text { No who } \\
\text { underwent } \\
\text { AF tests }\end{array}$ & $\begin{array}{l}\text { No with } \\
\text { abnormal } \\
\text { AFs }(\%)\end{array}$ \\
\hline Irritable bowel syndrome & 39 & 33 & $9(27)$ \\
\hline $\begin{array}{l}\text { Idiopathic upper gastro- } \\
\text { intestinal dysmotility }\end{array}$ & 23 & 21 & $6(29)$ \\
\hline Diabetes mellitus & 13 & 13 & $9(69)$ \\
\hline $\begin{array}{l}\text { Non-diabetic neurological } \\
\text { syndromes }\end{array}$ & 9 & 9 & $6(67)$ \\
\hline $\begin{array}{l}\text { After vagotomy/abdominal } \\
\text { surgery } \\
\text { Myopathic }\end{array}$ & $\begin{array}{l}17 \\
12\end{array}$ & $\begin{array}{r}11 \\
7\end{array}$ & $\begin{array}{l}3(27) \\
2(29)\end{array}$ \\
\hline
\end{tabular}

upper gastrointestinal dysmotility, was used to develop two models (one with just the motility score, and one with both motility and autonomic scores) for distinguishing neuropathic patients from all other patients evaluated. These models were then applied to the scores from idiopathic patients to estimate a predicted probability of neuropathic disorder for each idiopathic patient

A separate logistic discriminant analysis used both scores from all patients to determine if autonomic tests could add to the motility test's ability to discriminate irritable bowel syndrome patients from the other subgroups combined.

Receiver operating characteristic curves $\left(\mathrm{ROC}^{20}\right)$ were used to summarise the sensitivity and specificity of motility tests alone versus motility and autonomic function tests combined in addressing the two specific aims: identification of those with neurological disorders, and dif ferentiation of irritable bowel syndrome from the remaining patients.

\section{Results}

CLASSIFICATION OF PATIENTS AND PREVALENCE OF AUTONOMIC DYSFUNCTION

The following diagnostic categories were identified in 113 patients: irritable bowel syndrome $(\mathbf{n}=39)$; idiopathic upper gastrointestinal dysmotility $(n=23)$; diabetes mellitus $(n=13)$; identified non-diabetic neurological $(n=9)$ previous vagotomy or other abdominal surgery $(n=17) ;$ and myopathic pseudo-obstruction $(n=12)$. The specific diagnoses in each category are listed in Table III. The results of autonomic testing for each group are listed in Table IV. The relative motility and autonomic scores are shown in Figures 1 and 2. All patients with diabetes mellitus had autonomic function tests, and $64 \%$ of these patients had autonomic dysfunction. Three of four diabetic patients with normal autonomic function had normal gastrointestinal motility.

Among the remaining subgroups of patients, the prevalence of autonomic dysfunction ranged from $27-29 \%$.

\section{CORRELATION BETWEEN AUTONOMIC AND} MOTILITY SCORES

There was a significant $(\mathrm{p}<0.05)$ but modest $(\mathrm{r}=0.26)$ rank correlation between autonomic and motility scores in the entire group. In the subgroup of patients with diabetes mellitus, this correlation was stronger $(\mathrm{r}=0.68 ; \mathrm{p}=0.01)$; similarly, a significant correlation was noted in the combined group of patients with diabetes mellitus and non-diabetic neurological syndromes $(n=22 ; r=0.49 ; p<0.05)$.

\section{LOGISTIC DISCRIMINANT ANALYSIS}

The model based on data from patients with diabetes mellitus and identified neurological syndromes showed that at a specificity of $72 \%$, the addition of the autonomic function score to the motility score increased the sensitivity for identifying a neuropathic disturbance from 73 to only $76 \%$ (Fig 3 ). In this analysis the addition of the autonomic score changed the corresponding cut off in predicted probability of a neuropathic disturbance from 0.376 for the motility score alone to 0.394 for the combined motility and autonomic scores.

The second logistic discriminant model could distinguish irritable bowel syndrome from nonirritable bowel syndrome patients at a specificity of $80 \%$, with a sensitivity of $90 \%$. Once again, the autonomic function score did not increase the accuracy of the motility test to differentiate these two groups (Fig 4).

\section{Discussion}

Our study shows that autonomic dysfunction is frequent in patients with symptoms suggestive of gastrointestinal dysmotility when a neurological diagnosis is clinically identifiable, as listed in Table III. Of the remaining patients referred for evaluation of a clinically suspected motility disorder at a tertiary referral centre, the prevalence of visceral autonomic dysfunction is around $27-29 \%$, and is virtually the same in all diagnostic groups. Our study does not permit us to determine whether dysautonomia is of aetiological importance in patients with irritable bowel syndrome, idiopathic upper gastrointestinal dysmotility, and those with previous vagotomy or abdominal surgery. However, our findings confirm the frequent association of motility disorders with autonomic dysfunction. Other workers have reported vagal dysfunction of variable severity in patients with gastrooesophageal reflux. ${ }^{3+15}$ In our group of patients who had previously undergone vagotomy or abdominal surgery, the diagnosis of autonomic dysfunction was based on tests which evaluate 
thoracic and peripheral autonomic pathways that are unaffected by the abdominal surgery. It is possible that subtle autonomic dysfunction may have preceded the surgical treatment. Although functions of the infradiaphragmatic vagus nerve, such as the pancreatic polypeptide response to hypoglycaemia or sham feeding, ${ }^{16}$ were not evaluated in these studies, the tests used in this study are comprehensive. In fact, Buysschaert $e t$ $a l^{18}$ have previously shown that, in conditions such as diabetes mellitus, which are associated with a length dependent neuropathy, abnormal cardiovascular reflexes are an excellent predictor of dysfunction of the abdominal vagus.

Our data also suggest that autonomic dysfunction may result in gastrointestinal dysmotility of varying severity. Some of these dysmotilities are manifest clinically and on special testing (for example by manometry) as either irritable bowel syndrome or idiopathic upper gastrointestinal motility disorders. In some patients the symptoms may have been severe enough to lead to surgical treatment before referral to our clinic.

Read ${ }^{17}$ has elegantly indicated the difficulty in categorising the spectrum of more severely affected patients with apparently functional gastrointestinal disease. While intestinal manometric criteria may help make this distinction, ${ }^{8}$ the similar prevalence of dysautonomia found in our study provides support for Read's contention

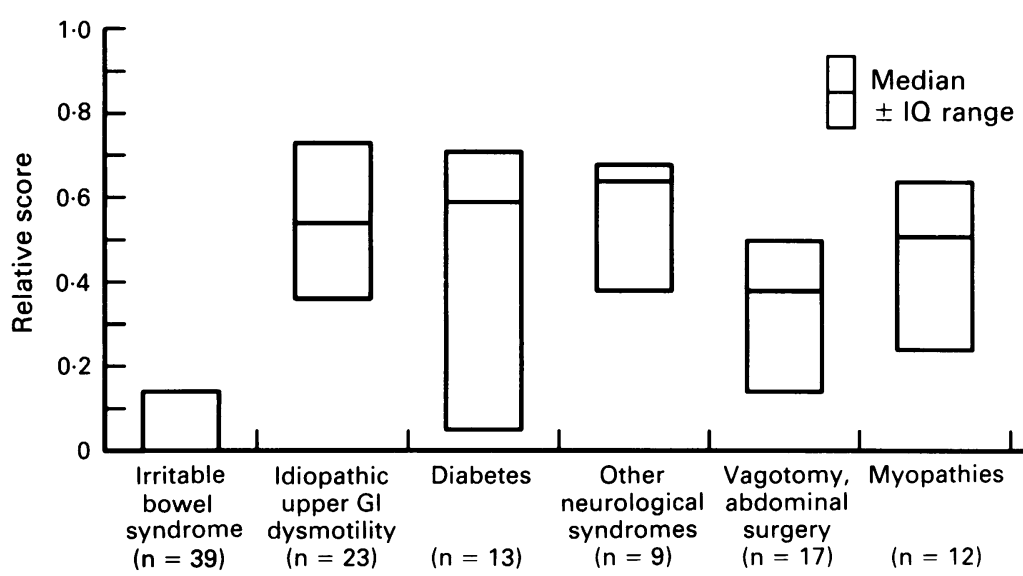

Figure 1: Distribution of gastrointestinal motility scores in different patient groups. Raw scores were computed by the system shown in Table I. Relative score were obtained from dividing the maximum possible for an individual patient.

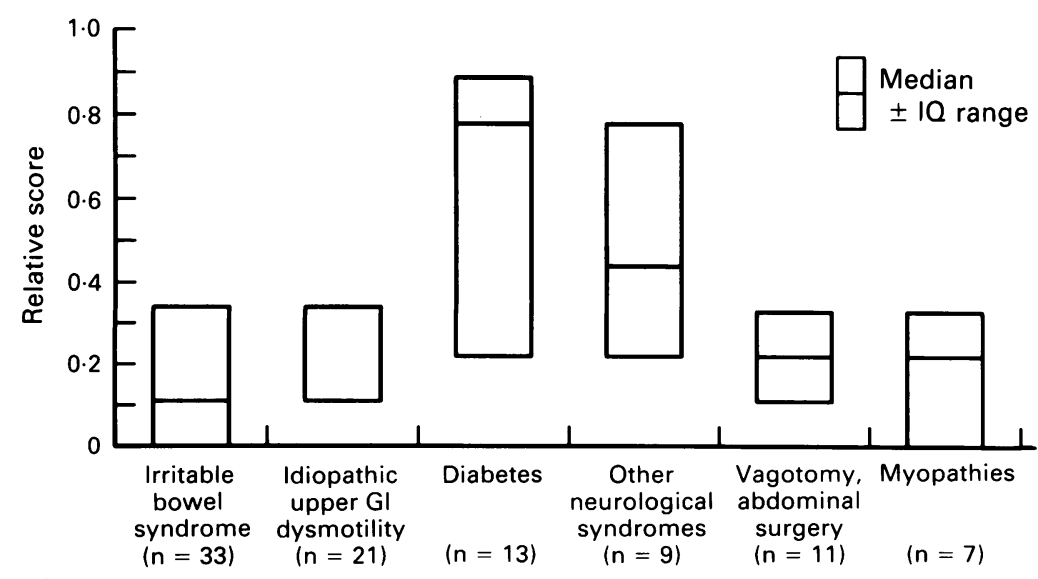

Figure 2: Distribution of autonomic function scores in different patient groups. Raw scores were computed by the system shown in Table II. A maximum score of 9 was used to obtain relative scores for each patient. that the terms irritable bowel syndrome and pseudo-obstruction may merely 'describe' different categories in the spectrum of functional gastrointestinal disease. ${ }^{17}$

The second major objective of our study was to evaluate the diagnostic utility of autonomic function testing. Based on the fact that a similar proportion of patients with irritable bowel syndrome, idiopathic upper gastrointestinal dysmotility, and previous surgery in our series had autonomic dysfunction one would correctly anticipate that these tests would not permit a clear distinction of the irritable bowel syndrome group from the rest. Indeed, the logistic discriminant models showed that autonomic tests did not add to the diagnostic value of the motility study. Among patients with diabetes and other identifiable neurological syndromes, autonomic tests were abnormal in 69\%, compared with the motility test which was abnormal in $73 \%$. Thus, if motility testing is not available, the non-invasive autonomic tests may be useful in confirming the presence of visceral denervation in those patients with symptoms suggestive of gut dysmotility. It must also be stressed that autonomic testing is extremely useful in assessing autonomic involvement outside the gastrointestinal tract.

Two patients with hollow visceral myopathy had abnormal vasomotor and sudomotor responses. The low amplitude fasting and postprandial intestinal pressure profiles suggested a myopathic disorder. Thus, the occurrence of visceral or vasomotor autonomic dysfunction does not necessarily imply that the gut dysmotility is the result of the neuropathic dysfunction.

Since only $27-29 \%$ of patients with idiopathic upper gastrointestinal dysmotility or postabdominal surgery have evidence of autonomic dysfunction, it is possible that disorders of the efferent extrinsic neurological pathways are not aetiologically important in these conditions. However, it is important to note that our data do not assess visceral afferent function or the enteric nervous system, which are presently believed to be at least partly responsible for the development of symptoms or abnormal motor function in the functional gastrointestinal syndromes. Among patients with previous vagotomy, it is likely that abdominal vagal denervation, which was not tested in our study, resulted in gastrointestinal dysmotility. ${ }^{9}$

Within the constraint dictated by a tertiary referral centre, we believe our study of 113 consecutive patients is unique since it encompasses all major subclasses of motility disorders from idiopathic dysmotility to gut motor dysfunction secondary to identifiable neurological syndromes. The standardisation of motility and autonomic tests and high proportion $(83 \%)$ of patients who underwent both tests allow estimates of the prevalence of autonomic dysfunction among patients referred to a centre specialising in gut motility disorders. Autonomic dysfunction is frequent among patients with identifiable neurological syndromes who present with gastrointestinal symptoms. Autonomic dysfunction of an idiopathic nature is present in a minority of patients with functional gastro- 


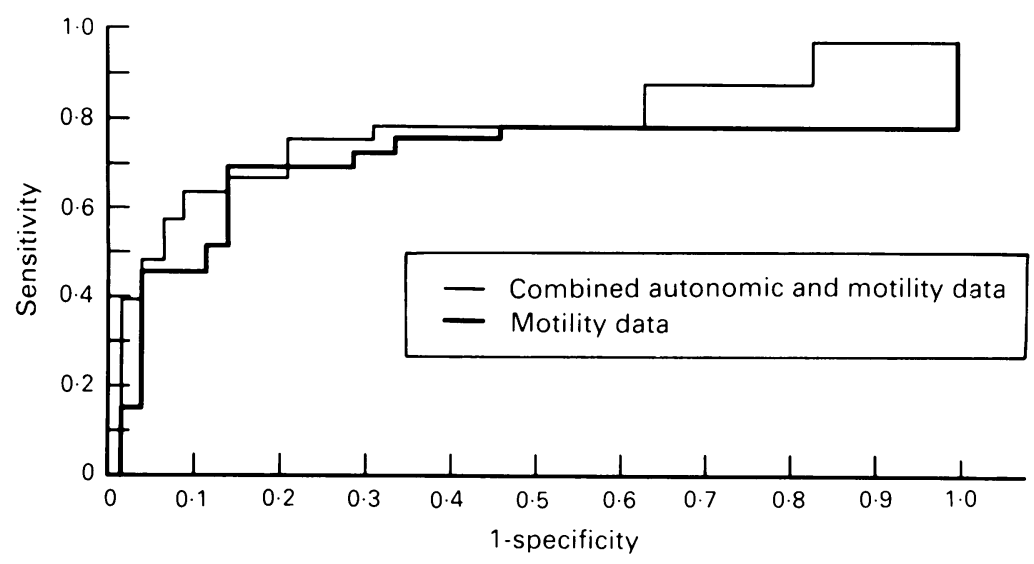

Figure 3: Receiver operating characteristic curve comparing accuracy in diagnosis of a neuropathic disorder of motility score alone and combined motility and autonomic function data based on logistic discriminant models. Note the similarity of curves suggesting autonomic data do not add to accuracy of the motility test.

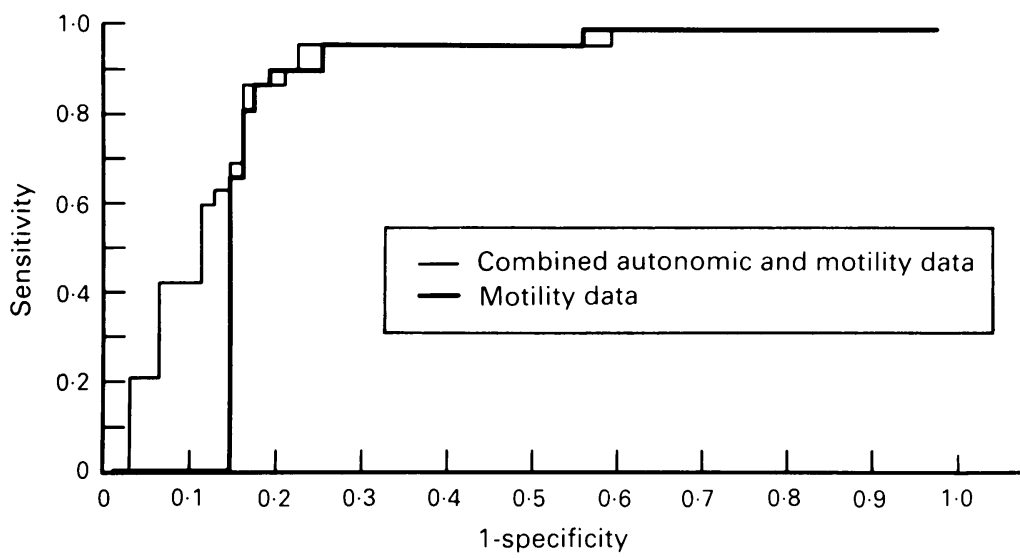

Figure 4: Receiver operating characteristic curve comparing accuracy in diagnosis of irritable bowel syndrome of motility score alone and combined motility and autonomic function data based on logistic models. The similarity of the curves indicates autonomic data do not add to the accuracy of the motility test. motility studies are not available or contraindicated.

This study was supported in part by the General Clinical Research Center grant RR00585 from the National Institute of Health. We thank Mrs Cindy Stanislav for typing and preparing this manuscript. This study was presented in part at the Annual Meeting of the American Gastroenterological Association in San Francisco, California, May 1992, and appears in abstract form in Gastroenterology 102: Ä425, 1992.

1 Marlett JA, Code CF. Effects of celiac and superior mesentric ganglionectomy on interdigestive myolectric complex in dogs. Am F Physiol 1979; 237: E432-6.

2 Camilleri $M$. Disorders of gastrointestinal motility in neurologic diseases. Mayo Clin Proc 1990; 65: 825-46.

3 Heatley RV, Collins RJ, James PD, Atkinson M. Vaga function in relation to gastro-oesophageal reflux and associated motility changes. BMF 1980; $280: 755-7$.

4 Chakraborty TK, Ogilvie AL, Heading RC, Ewing DJ Abnormal cardiovascular reflexes in patients with gastrooesophageal reflux. Gut 1989; 30: 46-9.

5 Camilleri $M$, Fealey RD. Idiopathic autonomic denervation in eight patients presenting with functional gastrointestinal eight patients presenting with functional gastrointestinal

6 Wood JD. Entric neurophysiology. Am $\mathcal{F}$ Physiol 1984; 247 G585-98.

7 Malagelada J-R, Camilleri M, Stanghellini V. Manometric diagnosis of gastrointestinal motility disorders. New York: Thieme Medical Publishers, 1986

8 Stanghellini V, Camilleri M, Malagelada J-R. Chronic idiopathic intestinal pseudo-obstruction: clinical and intestinal manometric findings. Gut 1987; 28: 5-12.

9 Fich A, Neri M, Camilleri M, Kelly KA, Phillips SF. Stasis syndromes following gastric surgery: clinical and motility features of sixty symptomatic patients. 7 Clin Gastroenterol 1990; 12: 505-12.

10 Summers RW, Anuras S, Green J. Jejunal manometry patterns in health, partial intestinal obstruction, and pseudo-obstruction. Gastroentrology 1983; 85: 1290-300.

11 Camilleri M. Jejunal manometry in distal subacute mechanical obstruction: significance of prolonged simultaneous conobstruction: significance of prolo

12 Mearin F, Camilleri M, Malagelada J-R. Pyloric dysfunction in diabetes with recurrent nausea and vomiting. Gastroenterology 1986; 90: 1919-25.

13 Kellow JE, Phillips SF. Altered small bowel motility in irritable bowel syndrome is correlated with symptom. Gastroenterology 1987; 92: 1885-93.

14 Kellow JE, Gill RC, Wingate DL. Prolonged ambulant recordings of small bowel motility demonstrate abnormalities in the irritable bowel syndrome. Gastroenterology 1990; 98: in the irritat

15 Cunningham KM, Horowitz M, Riddell PS, Maddern GJ Myers JC, Holloway RH, et al. Relations among autonomic nerve dysfunction, oesophageal motility, and gastric nerve dysfunction, oesophageal motility, and gastric emptying in

16 Schwartz TW, Holst JJ, Fahrenkrug J, Lindkaer Jensen S, Nielsen OV, Rehfeld JF, et al. Vagal, cholinergic regulation of pancreatic polypeptide secretion. 7 Clin Invest 1978; 61 : $781-9$.

intestinal disorders and may contribute to the aetiology in these patients. Finally, although autonomic testing adds little to the diagnostic accuracy of a gastrointestinal motility study in patients with a suspected motility disorder, they may contribute significantly to identifying autonomic involvement outside the digestive tract and to the diagnosis of patients with identified neurological syndromes and gut symptoms when 